Pacific Journal of Mathematic 


\title{
ON LIOUVILLE'S THEORY OF ELEMENTARY FUNCTIONS
}

\author{
MAXWELl ROSENLICHT
}

\begin{abstract}
Some recent results of $\mathrm{Ax}$ have made possible greatly simplified demonstrations of Liouville's basic results on the elementary integration of functions and the elementary solution of transcendental equations, together with their generalizations in various directions. An essentially self-contained exposition of this material is given here.
\end{abstract}

1. For the convenience of the reader, we provide in this section a succinct and somewhat simplified treatment of the necessary parts of Ax's paper [1].

Let $k \rightarrow K$ be a fixed homomorphism of commutative rings. (Thus $K$ is a $k$-algebra. In all our applications $K$ will be a field and $k$ a subfield, but we may as well begin with the extra generality.) If $M$ is a $K$-module, by a $k$-derivation of $K$ into $M$ is meant a $k$ linear map $D: K \rightarrow M$ such that $D(x y)=x(D y)+y(D x)$ for all $x, y \in K$. In such a situation we have $D x^{n}=n x^{n-1} D x$ for all $x \in K$ and all positive integers $n$; taking $x=1, n=2$, we get $D 1=0$, and hence $D$ vanishes on the image of $k$ in $K$. The $k$-derivations of $K$ into $M$ form a $K$-submodule $\operatorname{Der}_{k}(K, M)$ of $\operatorname{Hom}_{k}(K, M)$. A derivation on (or of) the ring $K$ is simply a $Z$-derivation of $K$ into $K$ (that is, we take $k=Z, K=M$ ). A derivation on an integral domain extends to a unique derivation on its field of quotients, by means of the equation $D(x / y)=(y D x-x D y) / y^{2}$.

Proposition 1. Let $k \rightarrow K$ be a homomorphism of commutative rings. Then there exists a $K$-module $\Omega_{K / k}$ and a $k$-derivation $d$ of $K$ into $\Omega_{K / k}$ such that for any $k$-derivation $D$ of $K$ into a $K$-module $M$ there exists a unique $K$-homomorphism $\Omega_{K / k} \rightarrow M$ which composed with d gives $D$.

This well-known result is most simply proved by trying for $\Omega_{K / k}$ the $K$-module $\Phi / \Psi$, where $\Phi$ is the free $K$-module generated by the symbols $\{\delta x\}_{x \in K}$ and $\Psi$ the $K$-submodule of $\Phi$ generated by all $\delta(x+y)-\delta x-\delta y$ and all $\delta(x y)-x \delta y-y \delta x$ for $x, y \in K$, and all $\delta x$ for $x$ in the image of $k$ in $K$, with $d$ the obvious composition with $\delta$, and noting that this works.

The pair $\left(\Omega_{K / k}, d\right)$, clearly unique to within isomorphism, is called the module of $k$-differentials of $K$. Each element of $\Omega_{K / k}$ can be written as a finite sum $\sum_{i} x_{i} d y_{i}$, with $x_{i}, y_{i} \in K$. For any $K$-module 
$M$, there is a natural $K$-module isomorphism between $\operatorname{Der}_{k}(K, M)$ and $\operatorname{Hom}_{K}\left(\Omega_{K / k}, M\right)$.

Proposition 2. Let $\iota: k \rightarrow K$ be a homomorphism of commutative rings, $D$ a derivation on $K$ such that there exists a map $D_{k}: k \rightarrow k$ such that $\iota D_{k}=D$ c. Then there exists a unique map $D^{1}: \Omega_{K / k} \rightarrow \Omega_{K / k}$ such that for all $\omega, \eta \in \Omega_{K / k}$ and all $f \in K$ we have $D^{1}(\omega+\eta)=$ $D^{1} \omega+D^{1} \eta, D^{1}(f \omega)=(D f) \omega+f\left(D^{1} \omega\right)$, and $D^{1}(d f)=d(D f)$.

Since each element of $\Omega_{K / k}$ is of the form $\Sigma_{i} x_{i} d y_{i}$, with $x_{i}, y_{i} \in K$, the uniqueness of $D^{1}$ is clear. To prove the existence of $D^{1}$ we first define an additive map $D^{\prime}$ on the free $K$-module $\Phi$ of the proof of Proposition 1 by setting $D^{\prime}\left(\Sigma_{i} x_{i} \delta y_{i}\right)=\Sigma_{i}\left(\left(D x_{i}\right) \delta y_{i}+x, \delta\left(D y_{i}\right)\right)$ and then note that $D^{\prime} \Psi \subset \Psi$, so that $D^{\prime}$ defines an induced map $D^{1}$ on $\Phi / \Psi$, which is isomorphic to $\Omega_{K / k}$. The verification that $D^{1}$ has the desired properties is straightforward.

From now on $K$ will be a field, usually of characteristic zero, $k$ a subfield of $K$.

Lemma. Let $k$ be a field, $K$ a separable algebraic extension field of $k$. Then any derivation of $k$ has a unique extension to a derivation of $K$.

This is another standard result. A proof may be found in [2, $\S 3]$, for example.

Proposition 3. Let $k \subset K$ be fields, $\left\{x_{\alpha}\right\}_{\alpha \in A}$ elements of $K$ that are algebraically independent over $k$ and such that $K$ is separably algebraic over $k\left(\left\{x_{\alpha}\right\}_{\alpha \in A}\right)$. Then $\left\{d x_{\alpha}\right\}_{\alpha \in A}$ is a K-basis for $\Omega_{K / k}$.

Each element of $K$ satisfies a separable polynomial equation with coefficients in the ring $k\left[\left\{x_{\alpha}\right\}_{\alpha \in A}\right]$, and by applying $d$ to these equations we see that $d K \subset \Sigma_{\alpha \in A} K d x_{\alpha}$. In other words, $\left\{d x_{\alpha}\right\}_{\alpha \in A}$ spans $\Omega_{K / k}$. To show that $\left\{d x_{\alpha}\right\}_{\alpha \in A}$ are linearly independent over $K$, we use the existence, for each $\beta \in A$, of a derivation $\partial / \partial x_{\beta}$ of $K$ which annuls $k$ and each $x_{\alpha}, \alpha \neq \beta$, and takes on the value 1 at $x_{\beta}$; the derivation $\partial / \partial x_{\beta}$ is first constructed for the ring $k\left[\left\{x_{\alpha}\right\}_{\alpha \in A}\right]$, then extended to its field of quotients $k\left(\left\{x_{\alpha}\right\}_{\alpha \in A}\right)$, and then to $K$, using the lemma.

COROLLARY. If $k \subset K \subset L$ are fields of characteristic zero, then the natural homomorphism $\Omega_{K / k} \rightarrow \Omega_{L / k}$ is injective.

The "natural homomorphism" is of course that of Proposition 1. Injectivity results from Proposition 3, noting that any transcendence 
base for $K / k$ is part of a transcendence base for $L / k$.

Proposition 4. Let $k \subset K$ be fields of characteristic zero, let $u_{1}, \cdots, u_{n}, v$ be elements of $K$, with $u_{1}, \cdots, u_{n}$ nonzero, and let $c_{1}, \cdots, c_{n}$ be elements of $k$ that are linearly independent over the natural numbers $\boldsymbol{Q}$. Then the element

$$
c_{1} \frac{d u_{1}}{u_{1}}+\cdots+c_{n} \frac{d u_{n}}{u_{n}}+d v
$$

of $\Omega_{K / k}$ is zero if and only if each $u_{1}, \cdots, u_{n}, v$ is algebraic over $k$.

The element $d v$ of $\Omega_{k(v) / k}$ is zero if and only if $v$ is algebraic over $k$, by Proposition 3. The Corollary implies that the element $d v$ of $\Omega_{K / k}$ is zero if and only if $v$ is algebraic over $k$. It remains only to prove that if $u_{1}$ is not algebraic over $k$ then $c_{1} d u_{1} / u_{1}+\cdots+$ $c_{n} d u_{n} / u_{n}+d v$ is nonzero. For this we may replace $K$, if necessary, by $k\left(u_{1}, \cdots, u_{n}, v\right)$, to reduce ourselves to the case where $K$ is a finite extension of $k$. Let $x_{1}, \cdots, x_{m}$ be a transcendence base for $K / k$, with $x_{1}=u_{1}$. Considering the natural homomorphism $\Omega_{K / k} \rightarrow$ $\Omega_{K / k\left(x_{2}, \cdots, x_{m}\right)}$ and replacing $k$ by $k\left(x_{2}, \cdots, x_{m}\right)$ if necessary, we see that we may suppose $K$ algebraic over $k\left(u_{1}\right)$. Enlarge $K$, as we may if necessary, so that $K$ is normal over $k\left(u_{1}\right)$. If $c_{1} d u_{1} / u_{1}+\cdots+$ $c_{n} d u_{n} / u_{n}+d v=0$ then for any $\sigma \in \operatorname{Aut}\left(K / k\left(u_{1}\right)\right)$ we have $c_{1} d \sigma u_{1} / \sigma u_{1}+$ $\cdots+c_{n} d \sigma u_{n} / \sigma u_{n}+d \sigma v=0$, and adding up over all $\sigma \in \operatorname{Aut}\left(K / k\left(u_{1}\right)\right)$ produces an equation similar to our original one, but with $c_{1}$ replaced by $\left[K: k\left(u_{1}\right)\right] c_{1}$, with the same $c_{2}, \cdots, c_{n}, u_{1}$, and with $u_{2}, \cdots, u_{n}, v$ replaced by elements of $k\left(u_{1}\right)$. We therefore have to show that $c_{1} d u_{1} / u_{1}+\cdots+c_{n} d u_{n} / u_{n}+d v$ is nonzero in the special case where $u_{2}, \cdots, u_{n}, v \in K=k\left(u_{1}\right)$, with $u_{1}$ transcendental over $k$. This fact follows immediately upon expressing each $u_{i}$ as a power product of monic irreducible elements of $k\left[u_{1}\right]$ and an element of $k$ and $v$ in its partial fraction form relative to $k\left[u_{1}\right]$, for we then get a noncancelling partial fraction term $d u_{1} / u_{1}$.

Proposition 5. Let $k \subset K$ be fields of characteristic zero, $D$ a derivation of $K$ such that $D k \subset k, C$ the field $\{x \in k: D x=0\}$, and $u$ and $t$ elements of $K$ that are algebraically dependent over $C$. Then in $\Omega_{K / k}$ we have $D^{1}(u d t)=d(u D t)$.

For $D^{1}(u d t)=(D u) d t+u d D t$, while $d(u D t)=(D t) d u+u d D t$, so that we have to show that $(D u) d t=(D t) d u$. Corresponding to parts of the inclusions $C \subset k \subset k(u, t) \subset K$ we have the homomorphisms $\Omega_{K / C} \rightarrow \Omega_{K / k}$ and $\Omega_{k(u, t) / k} \rightarrow \Omega_{K / k}$, so that we can reduce ourselves first to the case $k=C$ and next to the case $K=k(u, t)=C(u, t)$. In this 
case $D$ is a multiple of the derivation $\partial / \partial t$ of $C(u, t)$ and our proof reduces to the known equality $d u=(\partial u / \partial t) d t$.

Proposition 6. Let $k \subset K$ be fields, $\triangle$ a set of derivations of $K$ such that $D k \subset k$ for each $D \in \Delta$, and let $C$ be the field $\bigcap_{D \in\lrcorner} \operatorname{ker} D$. Suppose $\omega_{1}, \cdots, \omega_{n} \in \Omega_{K / k}$ are annulled by each $D^{1}$, for $D \in \Delta$. Then if $\omega_{1}, \cdots, \omega_{n}$ are linearly dependent over $K$ they are linearly dependent over $C$.

For suppose that there are $a_{1}, \cdots, a_{n} \in K$, not all zero, such that $a_{1} \omega_{1}+\cdots+a_{n} \omega_{n}=0$. Choose $a_{1}, \cdots, a_{n}$ so that the number of nonzero $a_{i}$ 's is minimal, and that one of them, say $a_{1}$, is 1 . For each $D \in \Delta$ we get $0=D^{\prime}\left(a_{1} \omega_{1}+\cdots+a_{n} \omega_{n}\right)=\left(D a_{1}\right) \omega_{1}+\cdots+\left(D a_{n}\right) \omega_{n}=$ $\left(D a_{2}\right) \omega_{2}+\cdots+\left(D a_{n}\right) \omega_{n}$. Since the number of nonzero $a_{i}$ 's was minimal and $a_{1}=1$, we get $D a_{2}=\cdots=D a_{n}=0$. Hence each $a_{i} \in C$.

2. We come now to the applications of the previous material to differential algebra. The reader interested in the earlier literature should consult the bibliographies of the references listed at the end.

In what follows, by a differential field will be meant a field $k$, together with an indexed family $\left\{D_{i}\right\}_{i \in I}$ of derivations of $k$. For simplicity, one speaks of "the differential field $k$ ", instead of "the differential field $\left\{k,\left\{\left(i, D_{\imath}\right)\right\}_{i \in I}\right\}$ ". The constants of the differential field $k$ are $\bigcap_{i \in I} \operatorname{ker} D_{i}$, a subfield of $k$. A differential extension field of $k$ is an extension field $K$ of $k$ together with a family of derivations $\left\{D_{i}^{\prime}\right\}_{i \in I}$ of $K$ indexed by the same set such that the restriction of each $D_{i}^{\prime}$ to $k$ is $D_{i}$.

THEOREM 1. Let $k$ be a differential field of characteristic zero, $K$ a differential extension field of $k$ with the same constants $C$. For each $i=1, \cdots, n$ and $j=1, \cdots, \nu$ let $c_{i j} \in C$ and let $v_{i}$ be an element of $K, u_{j}$ a nonzero element of $K$. Suppose that for each $i=1, \cdots, n$ and each given derivation $D$ of $K$ we have

$$
\sum_{j=1}^{\nu} c_{i j} \frac{D u_{j}}{u_{j}}+D v_{i} \in k
$$

Then either deg. $\operatorname{tr} . k\left(u_{1}, \cdots, u_{\nu}, v_{1}, \cdots, v_{n}\right) / k \geqq n$ or the $n$ elements of $\Omega_{K / k}$ given by $\sum_{j=1}^{\nu} c_{i j} d u_{j} / u_{j}+d v_{i}, i=1, \cdots n$, are linearly dependent over $C$.

Working in $\Omega_{K / k}$ and using Propositions 2 an 5, for each given derivation $D$ of $K$ and each $i=1, \cdots, n$ we obtain 


$$
D^{1}\left(\sum_{j=1}^{\nu} c_{i j} \frac{d u_{j}}{u_{j}}+d v_{i}\right)=d\left(\sum_{j=1}^{\nu} c_{i j} \frac{D u_{j}}{u_{j}}+D v_{i}\right)=0 .
$$

If the differentials $\sum_{j=1}^{\nu} c_{i j} d u_{j} / u_{j}+d v_{i}, i=1, \cdots, n$, of $\Omega_{K / k}$ are linearly independent over $C$, then by Proposition 6 they are also linearly independent over $K$. Hence the $n$ differentials $\sum_{j=1}^{\nu} c_{i j} d u_{j} / u_{j}+$ $d v_{i}$ of $\Omega_{k\left(u_{1}, \cdots, u_{\nu}, v_{1}, \cdots, v_{n}\right) / k}$ are linearly independent over $k\left(u_{1}, \cdots, u_{\nu}\right.$, $\left.v_{1}, \cdots, v_{n}\right)$. Since $\Omega_{k\left(u_{1}, \cdots, u_{\nu}, v_{1}, \cdots, v_{n}\right) k}$ is a vector space over $k\left(u_{1}, \cdots, u_{\nu}\right.$, $\left.v_{1}, \cdots, v_{n}\right)$ of dimension deg. $\operatorname{tr} . k\left(u_{1}, \cdots, u_{\nu}, v_{1}, \cdots, v_{n}\right) / k$, this latter number must be at least $n$.

CoROllary. Let $k$ be a differential field of characteristic zero, $K$ a differential extension field of $k$ with the same constants. Suppose that $u_{1}, \cdots, u_{n}, v_{1}, \cdots, v_{n} \in K$, with $u_{1}, \cdots, u_{n}$ nonzero, and that for each $i=1, \cdots, n$ and each given derivation $D$ of $K$ we have $D u_{i} / u_{i}+D v_{i} \in k$. Then either deg. $\operatorname{tr} . k\left(u_{1}, \cdots, u_{n}, v_{1}, \cdots, v_{n}\right) / k \geqq n$ or some linear combination of $v_{1}, \cdots, v_{n}$ with constant coefficients that are not all zero and some power product of $u_{1}, \cdots, u_{n}$ with exponents not all zero are algebraic over $k$.

This is a slight generalization of the main result Theorem 4 of [1]. To prove it, note that if the transcendence degree in question is not at least $n$ then there exist $\gamma_{1}, \cdots, \gamma_{n} \in C$, not all zero, such that

$$
\gamma_{1} \frac{d u_{1}}{u_{1}}+\cdots+\gamma_{n} \frac{d u_{n}}{u_{n}}+\gamma_{1} d v_{1}+\cdots+\gamma_{n} d v_{n}=0,
$$

choose a basis $c_{1}, \cdots, c_{r}$ for the vector space $\boldsymbol{Q} \gamma_{1}+\cdots+\boldsymbol{Q} \gamma_{n}$ so that each $\gamma_{i}$ can be written as $\gamma_{i}=\sum_{j=1}^{r} \nu_{\imath j} c_{j}$ with each $\nu_{\imath j} \in Z$, hence (using "logarithmic derivatives") rewrite the displayed equation as

$$
\sum_{j=1}^{r} c_{j} \frac{d\left(u_{1}^{\nu} 1 j \cdots u_{n}^{\nu}{ }^{\nu}\right)}{u_{1}^{\nu} j \cdots u_{n}^{\nu} n j}+d\left(\gamma_{1} v_{1}+\cdots+\gamma_{n} v_{n}\right)=0,
$$

and quote Proposition 4.

The next theorem generalizes the main result of [3], to which paper we refer for its applications to the question of elementary solutions of transcendental equations. The lemma is an immediate consequence of Theorem 1 .

Lemma. Let k be a differential field of characteristic zero, $K a$ differential extension field of $k$ having the same constants and such that deg. $\operatorname{tr} . K / k=1$. Then any two k-differentials of $K$ which can be written in the form $c_{1} d u_{1} / u_{1}+\cdots+c_{n} d u_{n} / u_{n}+d v$, where $c_{1}, \cdots, c_{n}, u_{1}, \cdots, u_{n}, v \in k, c_{1}, \cdots, c_{n}$ being constants, in such a way that for each given derivation $D$ of $K$ we have $c_{1} D u_{1} / u_{1}+\cdots+$ 
$c_{n} D u_{n} / u_{n}+D v \in k$, are linearly dependent over the subfield of constants.

THEOREM 2. Let $k$ be a differential field of characteristic zero, $K$ a differential extension field of $k$ with the same constants, with $K$ algebraic over $k(t)$ for some given $t \in K$. Suppose that $c_{1}, \cdots, c_{n}$ are constants of $k$ that are linearly independent over $\boldsymbol{Q}$, that $u_{1}, \cdots, u_{n}, v$ are elements of $K$, with $u_{1}, \cdots, u_{n}$ nonzero, and that for each given derivation $D$ of $K$ we have $\sum_{i=1}^{n} c_{i} D u_{i} / u_{i}+D v \in k$. If for each given derivation $D$ of $K$ we have $D t \in k$, then $u_{1}, \cdots, u_{n}$ are algebraic over $k$ and there exists a constant $c$ of $k$ such that $v$ - ct is algebraic over $k$. If for each given derivation $D$ of $K$ we have $D t / t \in k$, then $v$ is algebraic over $k$ and there are integers $\nu_{0}, \nu_{1}, \cdots, \nu_{n}$, with $\nu_{0} \neq 0$, such that each $u_{2}^{\nu} / t^{\nu} i$ is algebraic over $k$.

We may suppose $t$ transcendental over $k$, so that $d t \neq 0$. In either of the two cases, each $D t \in k$ or each $D t / t \in k$, the Lemma is applicable, and we have $c_{1} d u_{1} / u_{1}+\cdots+c_{n} d u_{n} / u_{n}+d v$ equal to either $c d t$ or $c d t / t$, for some constant $c$ of $k$. In the former case we have $c_{1} d u_{1} / u_{1}+\cdots+c_{n} d u_{n} / u_{n}+d(v-c t)=0$, and Proposition 4 tells us that $u_{1}, \cdots, u_{n}, v-c t$ are all algebraic over $k$. In the latter case Proposition 4 first implies the linear dependence of $c_{1}, c_{2}, \cdots, c_{n}$ over $\boldsymbol{Q}$, so that we can write $c=\left(\sum_{l=1}^{n} \nu_{\imath} c_{i}\right) / \nu_{0}$, for suitable integers $\nu_{0}, \nu_{1}, \cdots, \nu_{n}$, with $\nu_{0} \neq 0$, and so obtain

$$
c_{1} \frac{d\left(u_{1}^{\nu_{0}} / t^{\nu_{1}}\right)}{u_{1}^{\nu_{0}} / t^{\nu_{1}}}+\cdots+c_{n} \frac{d\left(u_{n}^{\nu_{0}} / t^{\nu_{n}}\right)}{u_{n}^{\nu_{0}} / t^{\nu_{n}}}+d\left(\nu_{0} v\right)=0 .
$$

A final application of Proposition 4 to this last equation completes the proof.

If $k$ is a differential field and $x, y \in k$, with $y \neq 0$, and the relation $D x=D y / y$ holds for each given derivation $D$ of $k$, we call $x$ a logarithm of $y$ or $y$ an exponential of $x$. A differential extension field of $k$ is called an elementary extension of $k$ if it is of the form $k\left(t_{1}, \cdots, t_{N}\right)$, where for each $i=1, \cdots, N, t_{i}$ is either a logarithm of an element of $k\left(t_{1}, \cdots, t_{2-1}\right)$, or an exponential of an element of $k\left(t_{1}, \cdots, t_{i-1}\right)$, or is algebraic over $k\left(t_{1}, \cdots, t_{i-1}\right)$. In this case note that each field $k\left(t_{1}, \cdots, t_{\imath-1}\right)$ is a differential extension field of $k$.

The following result generalizes Liouville's theorem on the elementary integrability of functions.

THEOREM 3. Let $k$ be a differential field of characteristic zero and for each given derivation $D$ of $k$ let $\alpha_{D} \in k$. Then there exists an elementary differential extension field of $k$ having the same 
constants and containing an element $y$ such that $D y=\alpha_{D}$ for each given derivation $D$ if and only if there are constants $c_{1}, \cdots, c_{n} \in k$ and elements $u_{1}, \cdots, u_{n}, v \in k$, such that for each given derivation $D$ we have

$$
\alpha_{D}=\sum_{i=1}^{n} c_{i} \frac{D u_{i}}{u_{i}}+D v .
$$

First suppose that there is a differential extension field $k_{(}\left(t_{1}, \cdots, t_{N}\right)$ of $k$ having the same constants, with each $t_{i}$ a logarithm or an exponential of an element of $k\left(t_{1}, \cdots, t_{i-1}\right)$, or algebraic over the latter field, that contains an element $y$ such that $D y=\alpha_{D}$ for each given derivation $D$. We shall prove by induction on $N$ that elements $c_{1}, \cdots, c_{n}, u_{1}, \cdots, u_{n}, v$ of $k$ exist as indicated. Since the case $N=0$ is trivial, we assume that $N>0$ and that the result holds for $N-1$. If we apply the $N-1$ case to the differential fields $k\left(t_{1}\right) \subset k\left(t_{1}, \cdots, t_{N}\right)$ we deduce immediately that there are constants $c_{1}, \cdots, c_{n}$ of $k$ and elements $u_{1}, \cdots, u_{n}, v$ of $k\left(t_{1}\right)$ such that for each given derivation $D$ we have $\alpha_{D}=\sum_{i=1}^{n} c_{i} D u_{i} / u_{i}+D v$. Thus we are reduced to proving only the rather general statement that if there is an element $t$ in a differential extension field of $k$ having the same constants as $k$ such that $t$ is a logarithm or an exponential of an element of $k$ or algebraic over $k$ and if there exist an integer $n$, constants $c_{1}, \cdots, c_{n}$ of $k$, and elements $u_{1}, \cdots, u_{n}, v$ of $k(t)$ such that $\alpha_{D}=\sum_{r=1}^{n} c_{i} D u_{i} / u_{i}+D v$ for each given derivation $D$, then such $n$ and $c_{1}, \cdots, c_{n}, u_{1}, \cdots, u_{n}, v$ can be found with the latter all in $k$. If $t$ is algebraic over $k$, we can assume $k(t)$ to be a normal extension of $k$. Then for each $\sigma \in \operatorname{Aut}(k(t) / k)$ we have $\alpha_{D}=\sum_{i=1}^{n} c_{i} D \sigma u_{i} / \sigma u_{i}+D \sigma v$ and summing over all $\sigma$ we get $[k(t): k] \alpha_{D}=\sum_{l=1}^{n} c_{i} D \Pi_{\sigma} \sigma u_{i} / \Pi_{\sigma} \sigma u_{i}+D \Sigma_{\sigma} \sigma v$, with each element $\Pi_{\sigma} \sigma u_{i}$ and $\Sigma_{\sigma} \sigma v$ in $k$. Thus we may assume $t$ transcendental over $k$. We claim that we may suppose $c_{1}, \cdots, c_{n}$ to be linearly independent over $\boldsymbol{Q}$. For if, say, $c_{n}$ depends linearly on $c_{1}, \cdots, c_{n-1}$ we write $c_{n}=\left(m_{1} c_{1}+\cdots+m_{n-1} c_{n-1}\right) / m$, with $m_{1}, \cdots, m_{n-1}, m \in \boldsymbol{Z}$, $m \neq 0$ and we obtain for each given derivation $D$ the equation $\alpha_{D}=\sum_{i=1}^{n-1}\left(c_{i} / m\right) D\left(u_{\imath}^{m} u_{n}^{m_{i}}\right) / u_{i}^{m} u_{n}^{m_{i}}+D v$, similar to what we had before but with smaller $n$. Therefore we may assume that $c_{1}, \cdots, c_{n}$ are linearly independent over $\boldsymbol{Q}$. If $t$ is a logarithm of an element of $k$, say $D t=D a / a$ for some $a \in k$ and each given derivation $D$, then it is an immediate consequence of Theorem 2 that $u_{1}, \cdots, u_{n} \in k$, while $v=c t+w$, for some constant $c$ and some $w \in k$, so that for each $D$ we have $\alpha_{D}=c_{1} D u_{1} / u_{1}+\cdots+c_{n} D u_{n} / u_{n}+c D a / a+D w$, a relation of the type desired, since all the terms here are in $k$. If $t$ is an exponential of an element of $k$, say $D t / t=D b$ for some $b \in k$ and each given derivation $D$, Theorem 2 tells us that $v \in k$ and there are integers $\nu_{0}, \nu_{1}, \cdots, \nu_{n}$, with $\nu_{0} \neq 0$, such that each $u_{i}^{\nu} / t^{\nu i} \in k$. 
Thus for each $D$ we have

$$
D u_{i} / u_{i}=\left(1 / \nu_{0}\right) D u_{\imath}^{\nu_{0}} / u_{i}^{\nu_{0} 0}=\left(1 / \nu_{0}\right) D\left(u_{\imath}^{\nu_{0}^{0}} / t^{\nu^{\nu}}\right) /\left(u_{2}^{\nu_{0}} / t^{\nu_{i}}\right)+\left(\nu_{i} / \nu_{0}\right) D t / t .
$$

Noting that $v$ and each $u_{i}^{\nu} / t^{\nu}$ are in $k$ and that $D t / t=D b$, with $b \in k$, we get an expression for each $\alpha_{D}$ of the type desired, with all terms in $k$. It therefore remains only to prove the converse of what we have shown so far, namely that if there exist constants $c_{1}, \cdots, c_{n}$ in $k$ and elements $u_{1}, \cdots, u_{n}, v$ of $k$ such that for each $D$ we have $\alpha_{D}=\sum_{i=1}^{n} c_{i} D u_{i} / u_{i}+D v$, then there is an element $y$ in some elementary extension field of $k$ having the same constants such that for each $D$ we have $D y=\alpha_{D}$. It suffices to prove that for each $i,\left\{D u_{i} / u_{i}\right\}$ can be integrated in turn, without introducing new constants. In other words, it remains to show that if $a \in k, a \neq 0$, then there exists a differential extension field $k(t)$ of $k$ having the same constants and such that $D t=D a / a$ for each given derivation $D$. To do this, take $t$ transcendental over $k$ and make $k(t)$ a differential extension field of $k$ by defining, for each given derivation $D$ of $k, D t=D a / a$. We are all done, unless it happens that $k(t)$ has a constant not in $k$. So suppose that $f / g$ is a constant in $k(t)$, with $f, g$ relatively prime elements of $k[t]$, not both in $k$, and $g$ monic. For each given derivation $D$ of $k$ we have $D(f / g)=0$, so that $g D f=f D g$. Now $D f, D g \in k[t]$, with degrees respectively $\leqq$ (degree of $f$ ), $<($ degree of $g$ ). Relative primeness implies $g \mid D g$, so that $D g=0$, hence also $D f=0$. Therefore there is a constant in $k[t]$ that is not in $k$. Say that $b_{0}, b_{1}, \cdots, b_{n} \in k, n>0, b_{0} \neq 0$, with $D\left(b_{0} t^{n}+b_{1} t^{n-1}+\cdots+b_{n}\right)=0$ for all $D$. Then

$$
\left(D b_{0}\right) t^{n}+\left(n b_{0} D a / a+D b_{1}\right) t^{n-1}+\cdots=0
$$

for all $D$. Therefore $b_{0}$ is a constant in $k$ and $D a / a=D\left(-b_{1} / n b_{0}\right)$. In this case $a$ has a logarithm in $k$ itself and we are done.

Added in proof. Another proof of the main part of this theorem is given in B. F. Caviness and M. Rothstein, "A Liouville theorem on integration in finite terms for line integrals," Communications in Algebra, 3 (1975), 781-795.

\section{REFERENCES}

1. J. Ax, On Schanuel's conjectures, Ann. of Math., 93 (1971), 252-268.

2. M. Rosenlicht, Integration in finite terms, Amer. Math. Monthly, 79 (1972), 963-972.

3. - On the explicit solvability of certain transcendental equations, Publ. Math., No. 36 (1969), 15-22.

Received September 20, 1974. Research supported by National Science Foundation grant number GP-37492X. 


\section{PACIFIC JOURNAL OF MATHEMATICS}

\section{EDITORS}

RICHARD ARENS (Managing Editor)

University of California

Los Angeles, California 90024

\section{R. A. Beaumont}

University of Washington

Seattle, Washington 98105
J. DugundjI

Department of Mathematics

University of Southern California

Los Angeles, California 90007

D. Gilbarg and J. Milgram

Stanford University

Stanford, California 94305

\section{ASSOCIATE EDITORS}
E. F. BECKENBACH
B. H. NeumanN
F. WOLF
K. YosHIDA

\section{SUPPORTING INSTITUTIONS}

\author{
UNIVERSITY OF BRITISH COLUMBIA \\ CALIFORNIA INSTITUTE OF TECHNOLOGY \\ UNIVERSITY OF CALIFORNIA \\ MONTANA STATE UNIVERSITY \\ UNIVERSITY OF NEVADA \\ NEW MEXICO STATE UNIVERSITY \\ OREGON STATE UNIVERSITY \\ UNIVERSITY OF OREGON \\ OSAKA UNIVERSITY
}

\author{
UNIVERSITY OF SOUTHERN CALIFORNIA \\ STANFORD UNIVERSITY \\ UNIVERSITY OF HAWAII \\ UNIVERSITY OF TOKYO \\ UNIVERSITY OF UTAH \\ WASHINGTON STATE UNIVERSITY \\ UNIVERSITY OF WASHINGTON \\ AMERICAN MATHEMATICAL SOCIETY
}

The Supporting Institutions listed above contribute to the cost of publication of this Journal, but they are not owners or publishers and have no responsibility for its content or policies.

Mathematical papers intended for publication in the Pacific Journal of Mathematics should be in typed form or offset-reproduced, (not dittoed), double spaced with large margins. Please do not use built up fractions in the text of your manuscript. You may however, use them in the displayed equations. Underline Greek letters in red, German in green, and script in blue. The first paragraph or two must be capable of being used separately as a synopsis of the entire paper. Items of the bibliography should not be cited there unless absolutely necessary, in which case they must be identified by author and Journal, rather than by item number. Manuscripts, in triplicate, may be sent to any one of the editors. Please classify according to the scheme of Math. Reviews, Index to Vol. 39. All other communications should be addressed to the managing editor, or Elaine Barth, University of California, Los Angeles, California, 90024.

The Pacific Journal of Mathematics expects the author's institution to pay page charges, and reserves the right to delay publication for nonpayment of charges in case of financial emergency.

100 reprints are provided free for each article, only if page charges have been substantially paid. Additional copies may be obtained at cost in multiples of 50 .

The Pacific Journal of Mathematics is issued monthly as of January 1966. Regular subscription rate: $\$ 72.00$ a year (6 Vols., 12 issues). Special rate: $\$ 36.00$ a year to individual members of supporting institutions.

Subscriptions, orders for back numbers, and changes of address should be sent to Pacific Journal of Mathematics, 103 Highland Boulevard, Berkeley, California, 94708.

PUBLISHED BY PACIFIC JOURNAL OF MATHEMATICS, A NON-PROFIT CORPORATION

Printed at Kokusai Bunken Insatsusha (International Academic Printing Co., Ltd.), 8-8, 3-chome, Takadanobaba, Shinjuku-ku, Tokyo 160, Japan. 


\section{Pacific Journal of Mathematics \\ Vol. 65, No. $2 \quad$ October, 1976}

Andrew Adler, Weak homomorphisms and invariants: an example .......... 293

Howard Anton and William J. Pervin, Separation axioms and metric-like

functions ............................................. 299

Ron C. Blei, Sidon partitions and p-Sidon sets .................... 307

T. J. Cheatham and J. R. Smith, Regular and semisimple modules ........... 315

Charles Edward Cleaver, Packing spheres in Orlicz spaces .............. 325

Le Baron O. Ferguson and Michael D. Rusk, Korovkin sets for an operator on a

space of continuous functions ............................. 337

Rudolf Fritsch, An approximation theorem for maps into Kan fibrations ....... 347

David Sexton Gilliam, Geometry and the Radon-Nikodym theorem in strict

Mackey convergence spaces .................................

William Hery, Maximal ideals in algebras of topological algebra valued

functions ...................................... 365

Alan Hopenwasser, The radical of a reflexive operator algebra ........... 375

Bruno Kramm, A characterization of Riemann algebras................. 393

Peter K. F. Kuhfittig, Fixed points of locally contractive and nonexpansive

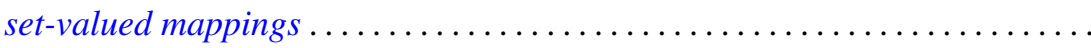

Stephen Allan McGrath, On almost everywhere convergence of Abel means of

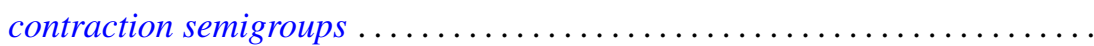

Edward Peter Merkes and Marion Wetzel, A geometric characterization of

indeterminate moment sequences............................ 409

John C. Morgan, II, The absolute Baire property ................... 421

Eli Aaron Passow and John A. Roulier, Negative theorems on generalized convex approximation .................................... 437

Louis Jackson Ratliff, Jr., A theorem on prime divisors of zero and characterizations of unmixed local domains ..............

Ellen Elizabeth Reed, A class of $T_{1}$-compactifications................... 471

Maxwell Alexander Rosenlicht, On Liouville's theory of elementary

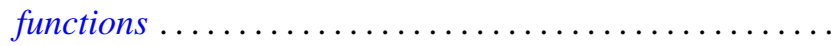

Arthur Argyle Sagle, Power-associative algebras and Riemannian

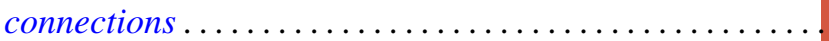

Chester Cornelius Seabury, On extending regular holomorphic maps from Stein manifolds...

Elias Sai Wan Shiu, Commutators and numerical ranges of powers of operators ...................................

Donald Mark Topkis, The structure of sublattices of the product of $n$ lattices ... 525

John Bason Wagoner, Delooping the continuous $K$-theory of a valuation

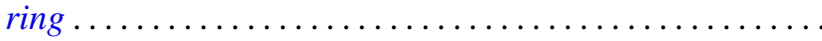

Ronson Joseph Warne, Standard regular semigroups...........

Anthony William Wickstead, The centraliser of $E \otimes_{\lambda} F \ldots$. 